Voix et Images

voixetimages

\title{
Vingt ans d'études québécoises au Brésil
}

\section{Zilá Bernd et Maria Bernadette Porto}

Volume 26, numéro 1 (76), automne 2000

\section{L’immonde}

URI : https://id.erudit.org/iderudit/201529ar

DOI : https://doi.org/10.7202/201529ar

Aller au sommaire du numéro

Éditeur(s)

Université du Québec à Montréal

ISSN

0318-9201 (imprimé)

1705-933X (numérique)

Découvrir la revue

Citer cet article

Bernd, Z. \& Porto, M. B. (2000). Vingt ans d'études québécoises au Brésil. Voix et Images, 26(1), 194-198. https://doi.org/10.7202/201529ar d'utilisation que vous pouvez consulter en ligne.

https://apropos.erudit.org/fr/usagers/politique-dutilisation/ 


\title{
Vingt ans d'études québécoises au Brésil
}

\author{
Zilá Bernd, Université fédérale du Rio Grande do Sul \\ Maria Bernadette Porto, Université fédérale Fluminense
}

L'intérêt pour les études québécoises au Brésil date de la fin des années soixante-dix lorsque le Ministère des Affaires internationales du Québec lança un programme de formation à l'intention des professeurs latino-américains. Cette initiative fut capitale et déterminante, car ce sont les professeurs universitaires de français qui, revenant du Québec, ont déclenché, un peu partout, des discussions, et proposé de mettre à l'étude ce qu'on appelait à l'époque les cultures et les littératures "d'expression française " dans l'enseignement universitaire au Brésil.

En effet, timidement au début, de manière plus enthousiaste voire agressive par la suite, les militants de la francophonie commencèrent à promouvoir, dans les programmes des universités fédérales, les littératures francophones d'Amérique, du Québec et des Antilles. Un des premiers à écrire sur ce sujet fut Italo Caroni de l'Université de São Paulo. De retour d'un stage au Québec, en 1978, il posa la question: quelle littérature doit-on enseigner au Brésil? Selon lui, il fallait d'abord "amplifier le concept de littérature, ouvrir des horizons plus vastes, offrir de nouvelles options à nos étudiants, la France n'étant pas le seul pays d'expression française ${ }^{1}$ ". Caroni souhaitait attirer un plus grand nombre d'étudiants vers le français et les litté- ratures de langue française, car, déjà à cette époque, l'attirance vers l'anglais commençait à se faire sentir. L'ouverture vers la francophonie se présentait comme une stratégie pour promouvoir le français, ce qui finalement ne s'est pas pleinement vérifié. Nous essaierons de le montrer.

À Rio de Janeiro, il faut souligner l'importance du rôle joué par Lilian Pestre de Almeida qui enseignait à l'Université fédérale Fluminense (UFF), ainsi qu'à l'Université fédérale de Rio de Janeiro (UFRJ). Ce fut surtout grâce à ses efforts que les littératures du Québec et des Antilles ont été introduites aux premier, deuxième et troisième cycles (Licenciatura, Mestrado et Doutorado). Outre la qualité du travail personnel de Lilian Pestre de Almeida, nous devons reconnaître sa participation dans la formation de jeunes chercheurs de l'époque, qui se sont tournés vers la francophonie. C'est le cas, parmi d'autres, d'Eurídice Figueiredo et de Maria Bernadette Porto - qui, en 1983, a soutenu la première thèse brésilienne en littérature québécoise, O espaço mítico em Kamouraska (L'espace mythique dans Kamouraska). Aujourd'hui, vingt ans plus tard, avec la collaboration d'autres collègues (Véra Lucia dos Reis et Vera Lúcia Soares), Maria Bernadette Porto et Eurídice Figueiredo ont développé un programme d'études 
francophones qui compte parmi les programmes les plus dynamiques et prisés au Brésil, avec des cours de spécialisation (360 heures destinées aux enseignants de lycée et aux étudiants qui ont terminé la Licence) et de Maîtrise en littératures francophones. Grâce au prestige de la francophonie dans ses cours aux études supérieures, l'UFF est reconnue pour son profil bien particulier dans l'ensemble des universités brésiliennes, et, à partir de 1994, le comparatisme établi entre la littérature brésilienne et les littératures francophones s'est encore renouvelé grâce à la création du cours de Doctorat en littérature comparée.

Si l'on veut tracer un bref historique des études québécoises au Brésil, il faut souligner le $\mathrm{V}^{\mathrm{e}}$ Congrès mondial de la FIPF, réalisé à Rio de Janeiro en 1981. De nombreux écrivains francophones y ont participé tels que Édouard Glissant, René Depestre et Anne Hébert, ainsi qu'un nombre représentatif de professeurs québécois comme Maximilien Laroche et Jean-Claude Castellain, entre autres. Parmi les thèmes de ce congrès, il y avait - pour la première fois dans un congrès brésilien "L'Amérique latine dialogue avec la francophonie" et "Pluralité linguistique et identité". La majorité des propositions soutenues par des chercheurs brésiliens présentaient des arguments enthousiastes pour l'introduction immédiate des littératures québécoise et antillaise dans le cadre de l'enseignement universitaire brésilien. Cette rencontre internationale a eu de fructueuses retombées et les années suivantes ont vu proliférer à l'Université fédérale Fluminense, à l'Université fédérale du Minas Gerais et à l'Université fédérale du Rio Grande do Sul, à Porto Alegre, grâce à l'initiative de Zilà Bernd, des propositions de cours et de séminaires portant sur les littératures et cultures francophones des Amériques. À la fin des années quatre-vingt, l'enthousiasme s'est accrû; la réalisation à Porto Alegre, en 1987, du VIII ${ }^{\mathrm{e}}$ Congrès national des professeurs de français en est la preuve. Ce congrès avait comme thématique centrale "Le français dans les Amériques" et a pu compter sur la participation d'un bon nombre de professeurs dont les communications portaient sur les littératures francophones, ce qui prouvait le bon état de santé de la francophonie au Brésil. On pouvait dès lors constater que la recherche, comme l'enseignement, avait atteint sa pleine maturité.

Si aujourd'hui, au début du troisième millénaire, l'état de l'enseignement du français au Brésil est un peu assombri par l'attrait exercé par l'anglais et l'espagnol - dans ce dernier cas, à cause du Mercosul -, la recherche, cependant, n'a cessé de croître, comme en atteste un nombre significatif de publications. Parmi les derniers lancements, signalons Fronteiras, passagens, paisagens na Literatura Canadense (Niterói, 2000), sous la direction de Maria Bernadette Porto, Identidades e estéticas compósitas (Porto Alegre, 1999), collectif dirigé par Zilá Bernd, Recortes transculturais (Niterói, 1998), édité par Eurídice Figueiredo et Eloína Santos et $A$ América francesa. Introdução à cultura quebequense qu'ont publié Nubia Hanciau et Sylvie Dion, en 1999, à Rio Grande. Une des caractéristiques de ces publications est la perspective comparatiste avec la 
littérature brésilienne et l'intérêt pour le phénomène récent des littératures migrantes dans le contexte québécois.

Si les traductions en portugais sont essentielles pour la visibilité de la littérature québécoise chez les lecteurs lusophones, il faudrait également signaler les publications à l'étranger qui démontrent la vitalité de la recherche brésilienne en littérature francophone et comparée, ainsi que la similarité des préoccupations entre chercheurs brésiliens et québécois. Parmi ces publications, mentionnons L'identitaire et le littéraire dans les Amériques, éditée à Québec (Nota Bene, 1999) par Bernard Andrès et Zilá Bernd et Confluences littéraires. Brésil/Québec: les bases d'une comparaison, publiée en 1992, à Montréal, par Michel Peterson et Zilá Bernd. Ces ouvrages présentent de nombreux articles de littérature comparée, ainsi qu'une bibliographie des "Travaux brésiliens en littérature québécoise et comparée (19701992)".

La constitution récente d'un groupe de travail, au sein de l'ANPOLL (Association nationale des professeurs de langues et littératures), réunissant des chercheurs de différentes universités brésiliennes en vue d'étudier les "Relations littéraires interaméricaines", atteste, de toute évidence, d'une croissance de la recherche francophone au Brésil. Un des volets privilégiés par cette recherche concerne les relations du Québec avec le Brésil. Des publications, des mémoires de Maîtrise et des thèses de Doctorat seront réalisés dans le cadre de ce projet.

La traduction littéraire, qui fait également partie de l'effort compara- tiste, a produit deux anthologies publiées par les presses de l'Université fédérale du Rio Grande do Sul, en 1991 (Vozes do Quebec) et en 1994 (Canadá, imagens de um país). Un projet de traduction de la production littéraire féminine du Québec est en train d'être mis sur pied par le groupe formé autour de Nubia Hanciau à la FURG (Fundação Universidade de Rio Grande).

Malgré les efforts du gouvernement canadien et du gouvernement québécois qui ont, entre autres, attribué des bourses de recherche à des professeurs et à des étudiants aux études su04périeures (comme celles distribuées par le Conseil international d'études canadiennes), muni nos bibliothèques d'importants dons de livres, fait la promotion du stage annuel qui continue à être réalisé à l'Université Laval encore aujourd'hui (après une interruption de quelques années) et dépêché, de façon systématique, des professeurs de différentes universités du Québec, les difficultés de consolidation de ce nouveau domaine ne sont pas négligeables car le nombre d'enseignants est - malgré notre programme de formation doctoral - encore insuffisant.

Lors des dix dernières années, grâce à l'initiative du Ministère des Affaires étrangères et du Commerce international (MAECI) et par l'entremise de l'Ambassade du Canada au Brésil, l'Association brésilienne d'études canadiennes (ABECAN) a été créée, et son travail est fortement appuyé par une dizaine de centres d'études canadiennes établis dans différentes universités brésiliennes. La revue Canadart, de l'ABECAN, a présenté jusqu'ici, dans chacun de 
ses dix numéros, des articles de chercheurs brésiliens et québécois qui s'intéressent aux études québécoises. À partir de l'an prochain, une nouvelle revue sera lancée: Interfaces Brésil/Canada qui sera également un précieux outil dans la diffusion des études québécoises et canadiennes au Brésil.

Un autre facteur qui a beaucoup contribué à la dynamisation de la culture et de la littérature québécoise au Brésil fut la signature de protocoles d'ententes inter-universitaires grâce auxquels des missions d'enseignement et de recherche ont été réalisées au cours des dix dernières années. Des accords institutionnels bilatéraux ont été, par la suite, signés avec l'UQÀM. Si, d'une part, les lettres québécoises sont diffusées au Brésil, les lettres brésiliennes ont trouvé une place dans les programmes de Lettres des universités québécoises. Des ouvrages collectifs ont été publiés au Brésil et au Québec, rendant compte d'une véritable réciprocité. Ce sont surtout les universités fédérales Fluminense et du Rio Grande do Sul qui se sont impliquées, de façon systématique, dans ces échanges qui fonctionnent depuis 1990, apportant d'un côté et de l'autre de fructueux résultats.

Si l'on constate, dans la plupart des universités brésiliennes, une baisse d'étudiants inscrits aux programmes d'études francophones, ce résultat, plutôt défavorable, est lié aux faibles possibilités offertes par le marché du travail, puisque le français a pratiquement été supprimé de l'enseignement secondaire au Brésil. Les possibilités limitées de débouchés professionnels empêchent l'épanouissement des études francophones au
Brésil, malgré le dynamisme qui ponctue la recherche universitaire.

En dépit des particularités de l'enseignement de la francophonie au sein de différentes régions brésiliennes, toutes ces expériences montrent que si l'enseignement du français (langue, culture, littérature) se poursuit encore au Brésil, c'est grâce aux études francophones à l'université. Si l'on peut prévoir un avenir pour le français au Brésil et en Amérique latine, cet avenir appartient à l'enseignement des littératures et des cultures francophones.

\section{$\stackrel{*}{* *}$}

À court terme, un colloque international intitulé "L'Américanité partagée ", qui se tiendra à Niterói (Rio de Janeiro) en novembre 2000, appuyé par l'AIEQ (Association internationale des études québécoises) et par l'Université fédérale Fluminense, contribuera à rendre plus visible la littérature québécoise au Brésil. Le $\mathrm{VI}^{\mathrm{e}}$ Congrès international de l'ABECAN (Association brésilienne d'études canadiennes), qui aura lieu en novembre 2001 à Porto Alegre, permettra également à un public plus large de prendre contact avec les études québécoises et canadiennes.

À moyen et à long terme, on verra : la publication de résultats d'un certain nombre de projets de recherche tel que CD-ROM. Anthologie de textes fondateurs pour une théorie de la littérature comparée interaméricaine, développé à l'Université fédérale du Rio Grande do Sul; le projet "Transferts culturels", développé au sein du CIEC (Conseil international d'études canadiennes), avec un volet 
consacré aux relations Québec/ Brésil; le projet "Un nouveau regard sur le paysage. Scènes et décor chez des auteurs de langue française en Amérique" de Véra Lucia dos Reis; les projets coordonnés par Maria Bernadette Porto, "La représentation du quotidien comme invention dans les littératures "périphériques" (Québec, Brésil, Antilles)" et "Babel revisitée: la construction d'une poétique des langues dans les Amériques "; un certain nombre de thèses de doctorat, comme celle de Nubia Hanciau sur le personnage de la sorcière dans les littératures des Amériques (Québec/Antilles) et celle d'Arnaldo Rosa Vianna Neto, centrée sur les traversées identitaires de l'espace américain dans les romans de Réjean Ducharme et de Nélida Piñon. Tous ces événements donnent une indication de la persistance des études québécoises au Brésil.

Pour clore ce bref panorama sur l'élaboration d'une tradition d'études québécoises au Brésil, il faudrait dire que, pour y réussir, des chercheurs brésiliens et québécois ont dû faire des efforts importants (matériels, géographiques, culturels) pour dialoguer à partir de leurs différences et échanger leurs expériences de membres issus de "collectivités neuves" (pour reprendre l'expression de Gérald Bouchard) en Amérique. Ainsi, à travers leurs spécificités, Brésiliens et Québécois multiplient les échanges culturels, partagent des pratiques plus ou moins parallèles, liées à des processus de constructions et de déconstructions identitaires. $\mathrm{Si}$, au début, l'appel de la francophonie s'annonçait plutôt comme une mode passagère aux yeux de certains, le choix des auteurs francophones de ceux du Québec en particulier s'impose de plus en plus comme un enrichissement au sein des réflexions critiques qui nous permettent d'approfondir les débats autour de notre propre projet identitaire.

1. Italo Caroni, "Reflexôes sobre o ensino da literatura francesa na universidade brasileira * Revista Lingua e Literatura, São Paulo, USP, $\mathrm{n}^{\circ} 7,1978$, p. 345-354. 